\title{
Fatores de Risco e Proteção Associados à Depressão Pós-Parto no Pré-Natal Psicológico
}

\author{
Alessandra da Rocha Arrais ${ }^{1}$ \\ ${ }^{1}$ Escola Superior de Ciências da Saúde, DF, Brasil. \\ Tereza Cristina Cavalcanti Ferreira de Araujo $^{2}$ \\ ${ }^{2}$ Universidade de Brasília, DF, Brasil. \\ Rafaela de Almeida Schiavo ${ }^{3}$ \\ ${ }^{3}$ Universidade Paulista, SP, Brasil.
}

\begin{abstract}
Resumo: Esta investigação teve por objetivos gerais: a) identificar fatores de risco e de proteção associados à Depressão Pós-Parto (DPP); e b) avaliar a contribuição do Pré-Natal Psicológico (PNP) como programa de prevenção em Saúde da Mulher. Para tanto, empreendeu-se uma pesquisa-ação organizada em três fases, reunindo um total de 198 gestantes. Na última fase, as participantes foram distribuídas em Grupo Intervenção - GI ( $n=47)$ e Grupo Controle - GC ( $n=$ 29). Para coleta de dados, utilizaram-se: Questionário Gestacional, BAI, BDI-II e EPDS. Os dados foram submetidos a análises estatísticas descritivas e comparativas. Não foi possível relacionar variáveis socioeconômicas, participação no PNP e desejo de gravidez com maior risco de DPP. Em contrapartida, verificou-se tal associação quanto a gravidez não planejada e a falta de apoio do pai do bebê. Contrariamente ao GC, não se constatou associação entre ansiedade e depressão gestacionais com a DPP no GI. Nesse grupo, $10,64 \%$ das puérperas revelaram tendência para DPP, ao passo que, em GC, 44,83\% mostraram tal propensão. Em suma, considera-se que o PNP, somado a fatores de proteção, constitui ação preventiva a ser desenvolvida pelo profissional de Psicologia no contexto do acompanhamento pré e pós-natal.
\end{abstract}

Palavras-chave: Depressão Pós-parto, Fatores de Risco, Fatores de Proteção, Prevenção, Pré-natal.

\section{Risk Factors and Protection Associated with Postpartum Depression in Psychological Prenatal Care}

\begin{abstract}
The general objectives of this research were: a) to identify risk and protection factors associated with Postpartum Depression (PPD); and b) to evaluate the contribution of Psychological Prenatal Care (PNP) as a prevention program in Women's Health. For this, an action research was organized in three phases, gathering a total of 198 pregnant women. In the last phase, the participants were divided into Intervention Group - GI $(n=47)$ and Control Group GC $(n=29)$. For data collection, we used: Gestational Questionnaire, BAI, BDI-II and EPDS. Data were submitted to descriptive and comparative statistical analyses. It was not possible to relate socioeconomic variables, participation in PNP and desire for pregnancy with a higher risk of PPD. In contrast, there was such association between unplanned pregnancy and lack of support from the baby's father. Contrary to GC, there was no association between gestational anxiety and depression with GD in GI. In this group, $10.64 \%$ of postpartum women showed a tendency for PPD, whereas in CG, $44.83 \%$ showed such propensity. In short, it is considered that PNP, together with protective factors, is a preventive action to be developed by the Psychology professional in the context of pre and postnatal follow-up.
\end{abstract}

Keywords: Postpartum Depression, Risk factors, Protection Factors, Prevention, Prenatal. 


\title{
Factores de Riesgo y Protección Asociados a la Depresión Post-Parto en el Prenatal Psicológico
}

\begin{abstract}
Resumen: Esta investigación tuvo por objetivos generales: a) identificar factores de riesgo y de protección asociados a la Depresión Post-Parto (DPP); y b) evaluar la contribución del Prenatal Psicológico (PNP) como programa de prevención en Salud de la Mujer. Para ello, se emprendió una investigación-acción organizada en tres fases, reuniendo un total de 198 mujeres embarazadas. En la última fase, las participantes fueron distribuidas en Grupo Intervención - GI ( $n=47)$ y Grupo Control - GC $(n=29)$. Para la recolección de datos, se utilizaron: Cuestionario Gestacional, BAI, BDI-II y EPDS. Los datos se sometieron a análisis estadísticos descriptivos y comparativos. No fue posible relacionar variables socioeconómicas, participación en el PNP y deseo de embarazo con mayor riesgo de DPP. En cambio, se verificó tal asociación en cuanto al embarazo no planificado y la falta de apoyo del padre del bebé. Contrariamente al GC, no se constató asociación entre ansiedad y depresión gestacionales con la DPP en el GI. En ese grupo, el 10,64\% de las puérperas revelaron tendencia a DPP, mientras que en GC, el 44,83\% mostró tal propensión. En suma, se considera que el PNP, sumado a factores de protección, constituye una acción preventiva a ser desarrollada por el profesional de Psicología en el contexto del seguimiento pre y postnatal.
\end{abstract}

Palabras clave: Depresión Post Parto, Factores de Riesgo, Factores de Protección, Prevención, Prenatal.

\section{Introdução}

Efetivamente, em Saúde Mental, muitos estudos já foram feitos sobre o puerpério, revelando o interesse dos estudiosos por esse período, em que a condição de 'tornar-se mãe' se imbrica com a vulnerabilidade humana e pode comportar riscos psiquiátricos. Entre os transtornos pesquisados, distinguem-se: o baby blues, o distúrbio de pânico pós-parto, as psicoses puerperais e a Depressão Pós-parto (DPP) (Cantillino, Zambaldi, Albuquerque, \& Sougey, 2010; Dois, Uribe, Villarroel, \& Contreras, 2012; Greinert, \& Milani, 2015; Miller, \& La Russo, 2011; Nunes, \& Phipps, 2013; Rojas et al., 2010; Sylvén, Ekselius, Sundstöm-Poromaa, \& Skalkidou, 2013; Vasconcellos, \& Teng, 2010).

Segundo o Manual Diagnóstico e Estatístico de Transtornos Mentais 5a Edição - DSM-V (APA, 2014), a DPP, foi incluída na seção sobre os Transtornos Depressivos e passou a se enquadrar como um especificador do Transtorno Depressivo Maior, compreendendo o período gestacional e as quatro semanas iniciais após o parto. Dentre os sintomas comuns estão os sentimentos de inutilidade ou culpa excessiva que podem ser delirantes, acentuada diminuição do interesse ou prazer. Cerca de $50 \%$ dos episódios depressivos maiores no pós-parto começam antes do parto propriamente dito, e por isso passaram a ser designados como episódios no periparto ou depressão perinatal na última edição do DSM (APA, 2014). Embora a literatura esclareça que os sintomas depressivos podem surgir em algum outro momento no primeiro ano de vida do bebê (Lucci, Otta, David, \& Chelini, 2016).

A DPP é uma condição prevalente que afeta globalmente as mulheres puérperas (Bottino, 2011). Se não tratada, aumenta o risco de suicídio materno e pode prejudicar a capacidade de parenting com efeitos adversos resultantes no desenvolvimento da prole (Carlesso, Souza, \& Moraes, 2014; Flores, Souza, Moraes, \& Beltrami, 2013; Goodman, 2007; Lima, \& Tsunechiro, 2008; Lucci et al., 2016; PoojaBansil et al., 2010).

A DPP é uma das complicações mais comuns vivenciadas durante o puerpério, e pode atingir $15 \%$ a $20 \%$ das puérperas em nível de população mundial (Garfield et al., 2015). No Brasil, os artigos têm apresentado percentagens ainda mais elevadas como os de Saraiva e Coutinho (2008) com percentual de $32 \%$ a $38 \%$ de DPP em brasileiras de baixa renda; e de Theme Filha, Ayers, Gama e Leal (2016) de 25\%, em média, no período de 6 a 18 meses após o nascimento do bebê.

Uma série de fatores influencia a vulnerabilidade ou a proteção de uma mulher com episódios depressivos no pós-parto (Miller, \& La Russo, 2011). O presente artigo apresenta e discute esses fatores de risco e proteção e a implementação de intervenções de prevenção primária, denominada de Pré-Natal Psicológico (PNP). 


\section{Fatores de risco e proteção associados à depressão pós-parto}

Fatores de risco são eventos ou situações já estabelecidas propícias ao surgimento de problemas físicos, psicológicos e sociais, que apresentam maior chance de surgir e maior intensidade no período gravídico-puerperal. Já os fatores de proteção são medidas preventivas, ou situações já estabelecidas que minimizem o impacto dos fatores de risco ou potencializam as influências positivas que transformam ou melhoram respostas pessoais (Calvetti, Muller, \& Nunes, 2007), no presente trabalho, no período da gravidez e do puerpério. De acordo com Figueira, Diniz e Silva Filho (2011), o estabelecimento de fatores de risco e proteção pode contribuir para melhor compreensão da doença e para a elaboração de estratégias de prevenção e de diagnóstico precoce da DPP.

Na revisão de literatura que foi a base para a presente investigação, observou-se que esses fatores têm sido amplamente discutidos na literatura mundial, normalmente agrupados em três categorias: A) Fatores de risco e proteção psicossociais; B) Fatores de risco e proteção sociodemográficos/contextual; e C) Fatores de risco e proteção físicos e suas respectivas subcategorias, apresentadas a seguir:

A.1) Fatores de risco psicológico/psiquiátrico: Ter histórico de episódios depressivos pessoais anteriores à gestação (Castro, Place, Billings, Rivera, Frongillo, 2015; Davey,Tough,Adair,\&Bezies,2011;El-Hachemetal.,2014; Figueiraetal.,2011;Martín-Santosetal.,2012;Moraisetal., 2015; Nunes, \& Phipps, 2013; Rodríguez, Pineda, Álvarez, \& Rojas, 2013; Rojas et al., 2010; Rohitkumar et al., 2014; Silva et al., 2012; Tian Tian et al., 2012; Urdaneta et al., 2011); Presença de estresse na gestação (Bos et al., 2013; Figueira et al., 2011; Lara, Navarro, Navarrete, \& Yle, 2010; Liu, \& Tronick, 2013; Martín-Santos et al., 2012; Nunes, \& Phipps, 2013; Rodrigues, \& Schiavo, 2011; Silva et al., 2012; Tian Tian et al., 2012); Ansiedade gestacional (Davey et al., 2011; Figueira et al., 2011; Garfield et al., 2015; Lara et al., 2010; Rogers, Kidokoro, Wallendorf, \& Inder, 2013); Depressão gestacional (Faisal-Cury, \& Menezes, 2012; McMahon et al., 2015; Nunes, \& Phipps, 2013; Turkcapar et al., 2015); História de DPP anterior (Faisal-Cury, \& Menezes, 2012; Kinsey, Baptiste-Roberts, Zhu, \& Kjerulff, 2014; Turkcapar et al., 2015); Presença de antecedentes psiquiátricos pessoais (Masmoudi et al., 2014; Turkcapar et al., 2015; Zambaldi, Cantilllino, Sougey, \& Rennó, 2010); Idealização da maternidade (Arrais, Mourão, \& Fragalle, 2014;
Greinert, \& Milani, 2015); Insatisfação com a gravidez (Rohitkumar et al., 2014; Turkcapar et al., 2015); e História familiar de transtornos mentais (Rojas et al., 2010; Zambaldi et al., 2010).

A.2) Fatores de proteção psicológico/psiquiátrico: Participar de um Programa de Pré-Natal com base numa abordagem psicológica (Arrais et al., 2014; Arrais, \&Araujo, 2016; Arrais, Lordello, \& Cavados, 2015; Collado, Saez, Favrod \& Hatem, 2014; Kozinszky et al., 2012); eteruma Relação/ligação saudáveldasgestantes com suas próprias mães (Bhandari et al., 2012; Miranda et al., 2012).

A.3) Fatores de risco suporte social/relações interpessoais: Baixo apoio social e familiar (Bos et al., 2013; Figueira et al., 2011; Guedes et al., 2011; Kathree, Selohilwe, Bana, \& Peterson, 2014; Martín-Santos et al., 2012; Nunes, \& Phipps, 2013; Rojas et al., 2010; Turkcapar et al., 2015; Urdaneta et al., 2011); Conflito e insatisfação conjugal (Guedes et al., 2011; Kathree et al., 2014; Lara et al., 2010; Masmoudi et al., 2014; Morais et al., 2015; Wu et al., 2014); Falta de apoio do parceiro (Guedes et al., 2011; Lara et al., 2010; Rodríguez et al., 2013; Silva et al., 2012; Urdaneta et al., 2011); Falta de apoio social no puerpério (Figueira et al., 2011; Kim, Connolly, \& Tamim, 2014; McMahon et al., 2015); Exposição à violência por parceiro íntimo no ciclo gravídico puerperal (Castro et al., 2015; Turkcapar et al., 2015) e Conflito familiar (Al Hinai, \& Al Hinai, 2014).

A.4) Fatores de proteção suporte social/relações interpessoais: Percepção de suporte social durante a gravidez (Konradt et al., 2011; Kozinszky et al., 2012); Apoio social no puerpério (Dantas, Araújo, Paulino, \& Maia, 2012); e Relações sociais positivas (Bhandari et al., 2012).

B.1) Fatores de risco e de proteção sociodemográficos/ contextual: Idade materna (Al Hinai, \& Al Hinai, 2014; Garfield et al., 2015; Janssen, Heaman, Urquila, O'Campo, \& Thiessen, 2011; Nunes, \& Phipps, 2013; Wu et al., 2014); Baixo status de escolaridade (Rodríguez et al., 2013; Tian Tian et al., 2012); Desemprego ou subemprego (Al Hinai, \& Al Hinai, 2014; Greinert, \& Milani, 2015; Tian Tian et al., 2012); Ser solteira (Garfield et al., 2015; Rogers et al., 2013; Zaconeta, Queiroz, Amato, Motta, \& Casulari, 2013).

B.2) Fatores de proteção sociodemográficos/contextual: Alto status de escolaridade (Urdaneta et al., 2011).

C.1) Fatores de risco físico/hormonal: História de síndromepré-menstrual(Moraisetal.,2015;Sylvénetal., 
2013; Tian Tian et al., 2012; Turkcapar et al., 2015); Desregulação hormonal e de citocinas inflamatórias (Okun et al., 2011); e da Anemia pós-parto precoce (Alharbi, \& Abdulghani, 2014).

C.2) Fatores de risco físico/obstétrico: Complicação/ intercorrência médica na gestação (Figueira et al., 2011; Urdaneta et al., 2011; Zambaldi et al., 2010); Gravidez não planejada (Castro et al., 2015; Rojas et al., 2010; Turkcapar et al., 2015); Gravidez indesejada (Kathree et al., 2014; Masmoudi et al., 2014; Nunes, \& Phipps, 2013); Parto cesariano (Urdaneta et al., 2011); História de aborto espontâneo (Kinsey et al., 2014; Zambaldi et al., 2010); Não amamentar até as oito semanas do pós-parto (Davey et al., 2011), Parto vaginal (Zambaldi et al., 2010); Falta de acompanhamento pré-natal ginecológico (Rodríguez et al., 2013); Problemas no parto atual (Rohitkumar et al., 2014), Intimate partner violence (IPV) moderada ou grave durante a gravidez (Castro et al., 2015).

C.3) Fatores de proteção obstétricos: Analgesia de parto (Ding, Wang, Qu, Chen, \& Zhu, 2014); Amamentação contínua (Ding et al., 2014) e Parto via vaginal (Urdaneta et al., 2011).

Com base na revisão de literatura de Aliane, Mamede e Furtado (2011), depreende-se que a DPP parece ser o resultado da interação de vários fatores de risco fisiológicos/hormonais, psicológicos e sociais. Isto sugere a importância da triagem para mulheres que têm esses fatores de risco para evitar a instalação dessa depressão. Esta detecção deve ser feita no início de pós-parto ou então nas consultas pré-natais anteriores. Isto permite um tratamento adequado para as mães, para a relação mãe-recém-nascido e, mais tarde, para o equilíbrio psicológico da criança. Portanto, o impacto na vida dos envolvidos requer um trabalho não só remediativo, mas também preventivo, a fim de evitar este grave transtorno.

Portanto, o conhecimento dos fatores de risco e de proteção da DPP é importante para o planejamento e execução de ações preventivas, como o PNP, uma vez que, a intervenção precoce, utilizando uma estratégia psicoterapêutica específica para gestantes, pode resultar em uma redução significativa na sintomatologia depressiva. Dessa forma, sabendo-se que a promoção da integridade biopsicossocial da gestante pode ser assegurada por meio de um acompanhamento cuidadoso das mães, o trabalho do PNP mostra-se como um recurso privilegiado de atenção integral à gestante e sua família (Arrais, \& Araujo, 2016).

\section{Pré-natal psicológico: Especificidades de um programa}

O PNP é uma intervenção grupal para gestantes insere-se em um contexto de prevenção de doenças psíquicas e promoção de saúde mental, por oportunizar espaço importante para que mães e pais exponham suas vivências, fantasias, medos, angústias, alegrias e tristezas advindas desse processo, e possam trocar experiências e construir juntos, a nova função parental. Bortoletti (2007) defende o espaço dialógico propiciado pela vivência grupal como fator de proteção na construção de estratégias que potencializam os processos ocorridos na maternidade.

Este tipo de pré-natal se propõe a prevenir situações adversas potencialmente decorrentes na gestação e no pós-parto, principalmente pelo seu caráter vivencial e interativo. Busca proporcionar as gestantes um entrosamento com outras mulheres que comungam sentimentos, sensações e emoções em um momento semelhante de suas vidas, porém vivido de forma idiossincrática por cada gestante ou casal (Arrais, \& Araújo, 2016). De acordo com Bortoletti (2007) a gestante tem benefícios com os grupos terapêuticos por poder compartilhar seu sofrimento com outras mulheres sob orientação profissional, ajudando, ainda, no diagnóstico precoce e tratamento, se for o caso. Assim, o PNP, além de instrumento preventivo em relação a doenças psíquicas como a DPP.

Os encontros têm por objetivo, acolher e dar voz às mulheres, informar, orientar e preparar cada uma delas para que passem por este processo da melhor maneira possível. Para tal, diversos temas são desenvolvidos ao longo do grupo, levando-se em consideração as características e necessidades do mesmo. Alguns dos temas que podem ser trabalhados: conceito de maternidade, mudanças na relação conjugal e familiar com a chegada do bebê, amamentação, tipos de parto etc. (Arrais, \& Araujo, 2016; Bortoletti, 2007).

Vê-se, portanto, que ambos os tipos de pré-natal, o ginecológico e o psicológico, são complementares já que têm por finalidade primordial buscar formas para melhorar o momento do parto e a vivência da maternidade e da paternidade, favorecendo o desenvolvimento saudável do bebê ao oportunizar um projeto de parentalidade que inclua aspectos educativos e psicodinâmicos (Arrais, \& Araújo, 2016).

Diante do exposto, esta investigação teve por objetivos: a) identificar fatores de risco e de proteção 
associados à DPP; e b) avaliar a contribuição do PNP como programa de prevenção em Saúde da Mulher.

\section{Método}

Delineamento: trata-se de uma pesquisa longitudinal, de curta duração, com delineamento baseado na metodologia da Pesquisa-Ação (Thiollent, 2008) e que ocorreu em três fases: $1^{\text {a }}$ fase: rastreamento dos fatores de risco e de proteção associados à DPP presente na amostra inicial; $2^{\text {a }}$ fase: proposta de intervenção e implantação do PNP, apenas para participantes do PNP; e $3^{\text {a }}$ fase: rastreio da DPP para a amostra final.

Contexto e participantes: 198 gestantes foram incluídas na $1^{\text {a }}$ fase da pesquisa. Elas estavam no $3^{\circ}$ trimestre da gestação e internadas em uma maternidade pública de Brasília considerada referência regional no atendimento à clientela de baixa renda e gestantes de alto risco. $\mathrm{Na} 2^{\mathrm{a}}$ fase apenas 47 gestantes da amostra total participaram do PNP e, na $3^{\text {a }}$ fase da pesquisa, apenas 76 das colaboradoras se mantiveram no estudo e formaram a amostra final, foram distribuídas em dois grupos de análise: 47 que participaram do PNP e formaram o Grupo Intervenção (GI) e 29 que não aderiram ao PNP e formaram o Grupo Controle (GC).

Instrumentos: Foi utilizado para a coleta de dados para caracterização das colaboradoras o Questionário Gestacional, elaborado pela pesquisadora (Arrais et al., 2015), sobre dados sociodemográficos, história da gestação, rede social de apoio e história de gestações anteriores, que teve por intuito identificar e rastrear os fatores de risco e proteção para a DPP presentes na amostra inicial. Aplicado na $1^{\text {a }}$ fase para as 198 colaboradoras.

Para rastrear especificamente os sintomas de ansiedade e depressão na gestação utilizaram-se o Inventário Beck de Depressão - BDI-II (Beck, Steer, \& Brown, 1996) e o Inventário Beck de Ansiedade - BAI (Beck, \& Steer, 1993). O BDI-II é uma escala composta por 21 itens com diferentes alternativas a respeito de como o sujeito tem se sentido nas duas últimas semanas, os quais correspondem a diferentes níveis de gravidade da depressão: mínima (0-13 pontos), leve (1419 pontos), moderada (20-28 pontos) e severa (29-63 pontos). Já o BAI consiste em 21 questões sobre como o sujeito tem se sentido na última semana, expressas em sintomas comuns de ansiedade, os quais a participante deve responder conforme seu recente estado mental, podendo no escore corresponder à ansiedade mínima (0-7 pontos), leve (8-15 pontos), moderada
(16-25) e severa (26-63 pontos). Utilizado na $1^{\text {a }}$ fase para ambos os grupos GI e GC.

Para avaliar sintomas de DPP utilizou-se a Escala de Depressão Pós-Parto de Edimburgo (EPDS) - foi elaborada na Grã-Bretanha, por Cox e Holden (2003), é o primeiro instrumento, encontrado na literatura, proposto para rastrear especificamente a DPP. É um instrumento de autorregistro, com dez questões de sintomas comuns de depressão e utiliza um formato de respostas do tipo likert com 35 itens que descrevem como uma mãe está se sentindo, após o nascimento de seu bebê, na última semana. Esta escala possui tradução para 11 idiomas e foi submetida a estudos de validade em vários países, inclusive no Brasil, por Santos, Martins e Pasquali (1999) e por Cantillino et al. (2010). Consiste no instrumento mais utilizado em estudos sobre DPP, segundo autores que fizeram revisões de literatura científica sobre a DPP tais como: Aliane et al. (2011), Galvão, Silva Júnior, Lima e Monteiro (2015) e Saraiva e Coutinho (2008). De acordo com o estudo de validação no Brasil, o melhor ponto de corte para rastreamento foi para escores maiores ou iguais a 12 , com sensibilidade de $82,6 \%$ (Zaconeta et al., 2013). Essas escalas foram aplicadas às 76 gestantes de ambos os grupos, GI e GC.

Procedimentos de coleta de dados: as colaboradoras da assinaram o Termo de Consentimento Livre e Esclarecido (TCLE). Todas essas mulheres foram convidadas a participar das sessões de PNP, que ocorreram na própria maternidade, uma vez por semana, com duração de três horas, cada. As que aceitaram o convite e compareceram a pelo menos três sessões, receberam informações a respeito dos tipos de parto, acompanhante na sala de parto, distúrbios emocionais do puerpério, ajuda qualificada no pós-parto, papel dos avós, amamentação, desmitificação da maternidade e paternidade. As que aceitaram colaborar com a pesquisa, mas não quiseram participar das sessões do PNP, permaneciam apenas em atendimento psicológico individual previsto na rotina de seu acompanhamento hospitalar, realizado pela psicóloga do setor. A $3^{\text {a }}$ fase da pesquisa aconteceu até três meses depois do parto.

A pesquisadora e seus bolsistas de iniciação científica (IC) monitoravam as gestantes e após da data prevista do parto entravam em contato, via telefone, para solicitar uma visita domiciliar para avaliar a presença de possíveis sintomas de DPP. Cabe ressaltar que nesta última fase da pesquisa houve uma redu- 
ção do número de colaboradoras da pesquisa, pois no período pós-parto muitas puérperas não foram localizadas, por erro nos dados de identificação, mudança de telefones ou endereços, retorno para sua cidade de origem em outro Estado, e outras não quiseram receber a visita, especialmente as que não participaram do PNP. Assim, a amostra no final da $3^{\text {a }}$ fase do estudo foi composta por 76 mulheres que foram avaliadas tanto durante a gestação, como depois, no pós-parto.

Estas 76 mulheres foram divididas em dois grupos da pesquisa: as que participaram do PNP e formaram o Grupo de Intervenção (GI) $(\mathrm{n}=47)$ e as que não participaram no PNP e compuseram o Grupo Controle (GC) ( $\mathrm{n}=29)$, e foram avaliadas duas vezes: durante a gravidez (T1) e até três meses após o parto (T2), conforme ilustra a Figura 1.

Procedimentos de análise de dados: Primeiramente, identificaram-se os fatores de risco e proteção presentes na amostra. Posteriormente, foi testada a associação da DPP com os dados socioeconômicos, os fatores de risco e de proteção do GI e do GC. Para tanto, os dados foram analisados usando o programa estatístico Programa $\mathrm{R}^{\circledR}$. Realizaram-se análises estatísticas descritivas e comparativas para ambos os grupos. A fim de verificar associação entre os fatores de risco e proteção rastreados com sintomas de DPP, utilizaram-se os testes Teste T, Q-quadrado, Teste Exato de Fisher e o Método de Monte Carlo, adotando-se como nível de significância $\mathrm{p}<0,05$.

Cabe esclarecer que o conteúdo das sessões do PNP, correspondente à $2^{\text {a }}$ fase da pesquisa, não foi alvo de análise para o presente artigo, pois o seu objetivo não foi avaliar a intervenção em si, mas identificar os fatores de risco e proteção associados à DPP e comparar a sua ocorrência de DPP entre os grupos GI e GC no puerpério, relacionando-os.

Procedimentos éticos: Este estudo foi aprovado pelo Comitê de Ética Em Pesquisa - FEPECS/SES-DF, sob o número CAAE 40402114.1.0000.5553, Parecer no 979.401 , de 23/02/2015. Esta pesquisa seguiu os princípios éticos quanto à proteção dos direitos, bem-estar e dignidade das colaboradoras, de acordo com Resolução no 466/12 do Conselho Nacional de Saúde, além de estar em consonância com os princípios do Código de Ética dos Psicólogos vigente (CFP, 2005).

\section{Resultados}

Resultados da $1^{\text {a }}$ Fase da pesquisa: identificação dos fatores de risco e proteção associados à DPP
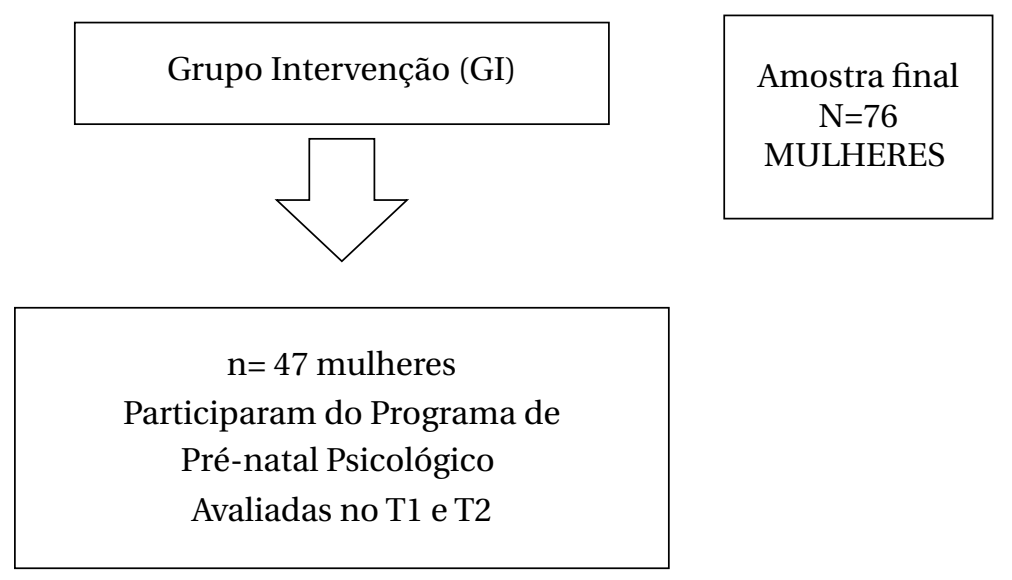

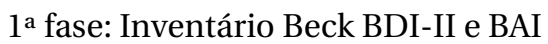

$2^{\text {a }}$ fase: Sessões do PNP

$3^{\text {a }}$ fase: Escala EPDS

Figura 1

Síntese dos procedimentos de coleta de dados.

PNP: pré-natal psicológico; EPDS: Edinburgh postnatal depression scale.
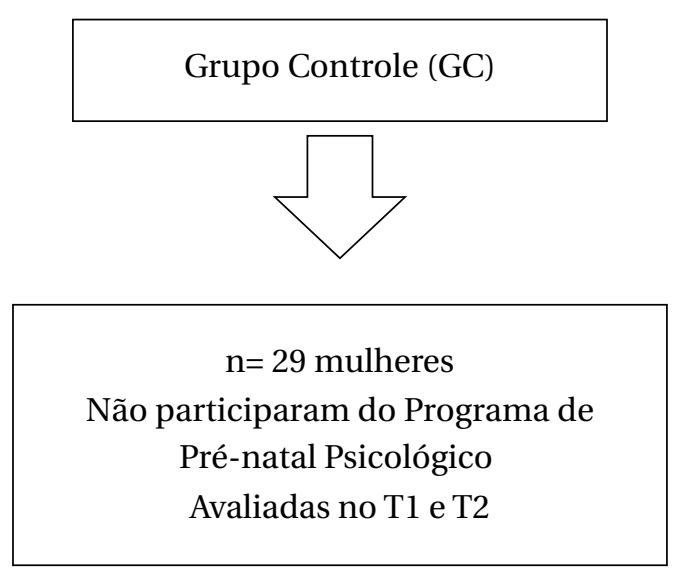

$1^{\text {a }}$ fase: Inventário Beck BDI-II e BAI $3^{\mathrm{a}}$ fase: Escala EPDS 
Inicialmente, analisou-se a frequência das variáveis consideradas pela literatura como de risco e proteção nas respostas das 198 colaboradoras ao questionário gestacional, quando se identificaram 23 fatores de risco e 11 fatores de proteção associados à DPP.

A Figura 2 apresenta os 23 fatores de risco para sintomas de DPP rastreados na amostra. Nota-se que o indicador que mais se mostrou frequente entre participantes desse estudo foi "ter passado por intercorrências na gravidez anterior/atual” (55), e em seguida aparece como o segundo indicador mais frequente "a cirurgia cesariana" (47). Observa-se ainda que fortes indicadores de risco para DPP como "não planejamento e desejo da gravidez", "ansiedade e depressão gestacional", "dificuldades financeiras", "rede de apoio empobrecida", "conflito familiar/conjugal" e "história de aborto" apresentaram uma frequência baixa nas respostas das participantes desse estudo.

Quanto aos 11 fatores de proteção para DPP identificados na amostra, observa-se que os mais frequentes foram: "ter suporte familiar" (63), "viver junto com o parceiro" (57), "ter desejado a gravidez" e "ter recebido apoio do pai da criança" (55), "não ter dificuldades financeiras" (52), "ter participado do PNP" (46), "teve um acompanhante na sala de parto" (44) e "ter relação conjugal satisfatória” (41).
Assim, em função dos fatores de risco e proteção identificados, nessa investigação, 18 ou $23,68 \%$ das mães foram identificadas como apresentando maior chance de apresentarem DPP, em ambos os grupos da pesquisa, o que caracteriza uma alta prevalência para risco de DPP na amostra investigada.

\section{Resultados da $3^{a}$ fase da pesquisa: análises comparativas entre GI e GC}

Os dados sociodemográficos e histórico obstétrico das colaboradoras colhidos por meio do questionário gestacional podem ser visualizados na Tabela 1 , e mostrou os resultados seguintes.

No GI a idade das gestantes variou entre 17 e 45 anos, com uma média de 29,10 anos e no GC o intervalo da idade esteve entre 15 e 41 anos, com a média de 28,5 anos. Todas estavam no terceiro trimestre da gestação. Nota-se, na Tabela 1, que a maioria das participantes do GI era casada, cursou ensino médio completo, estava empregada, tinha renda familiar de até $\mathrm{R} \$ 1.000,00$, era primigesta e católica. Já no GC, identificou-se diferença apenas nas variáveis escolaridade e renda familiar, pois a maioria tinha ensino superior e viviam com até $\mathrm{R} \$ 3.000,00$. Portanto, os grupos GI e GC eram semelhantes entre si com relação aos dados sociodemográficos.

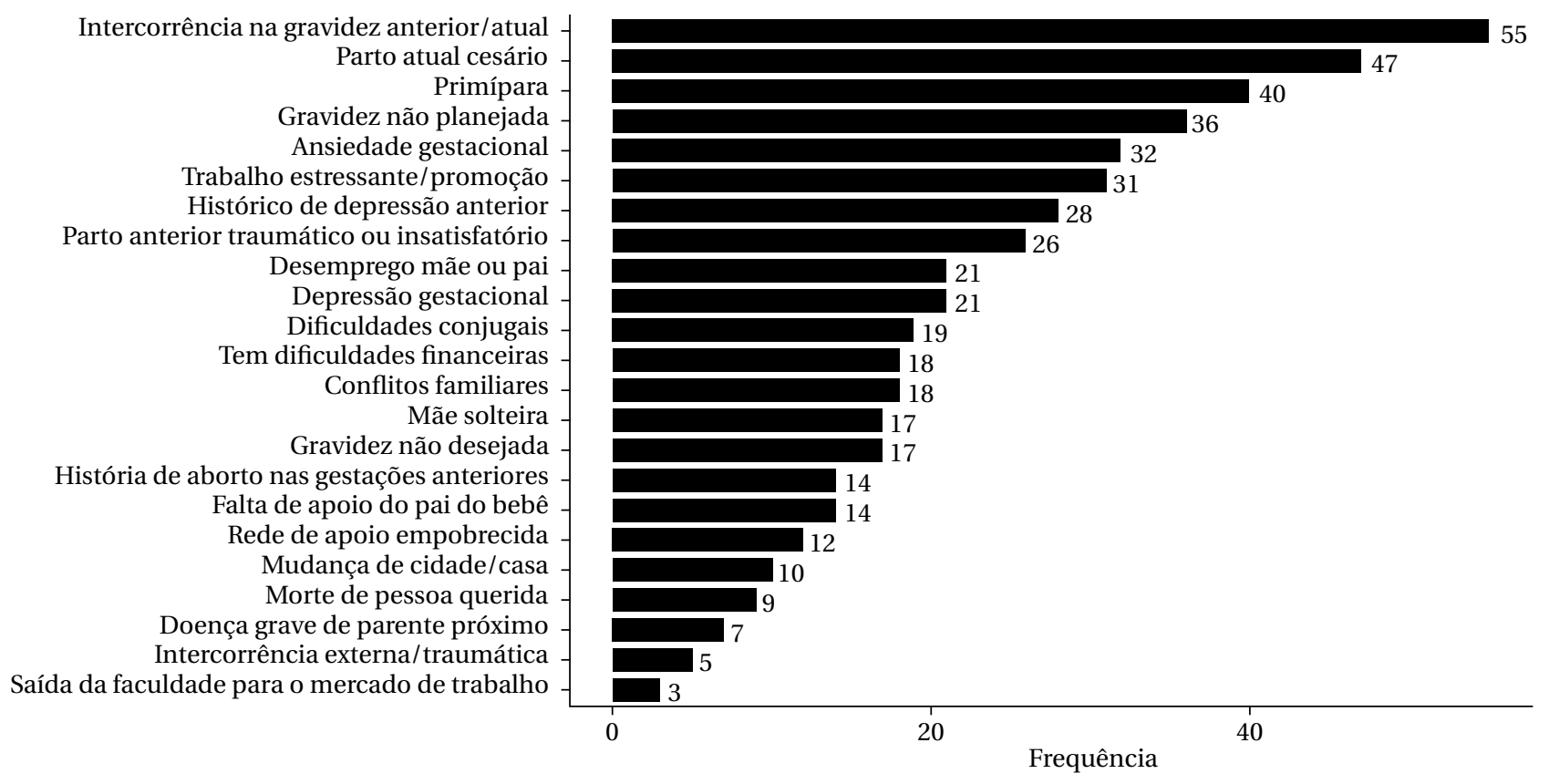

Figura 2

Frequência de mulheres com algum indicador de risco para DPP $(n=76)$. 


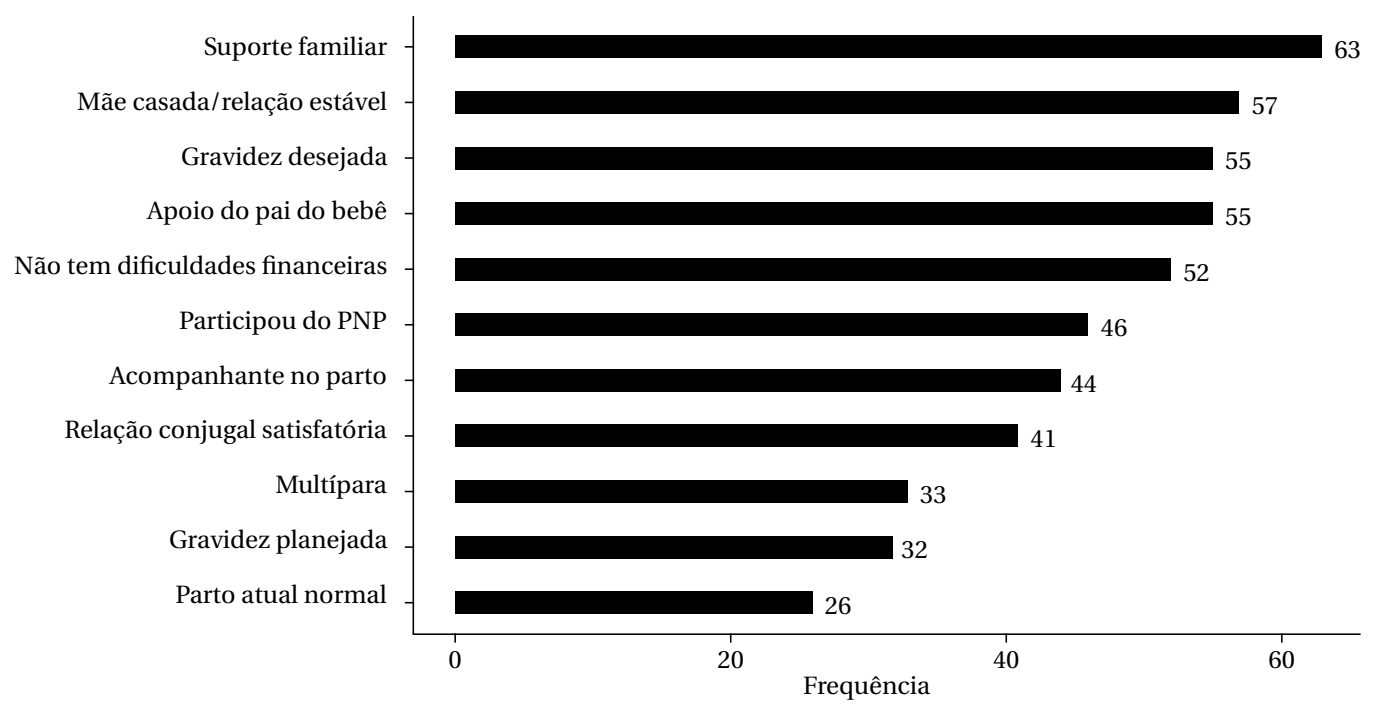

Figura 3

Frequência de mulheres com algum indicador considerado de proteção para DPP ( $n=76)$.

A fim de identificar fatores socioeconômicos que estão associados à DPP, realizaram-se testes de associação com as variáveis: idade, religião, escolaridade, renda familiar e número de gestações. Ao proceder à análise estatística de associação, entre os dados do GI e do GC verificou-se que não há associação entre as variáveis sociodemográficas e sintomas de DPP para as participantes deste estudo, conforme pode ser visualizado na Tabela 2.

Em contrapartida, ao proceder aos testes de associação entre os fatores de risco identificados nos grupos GI e GC, constatou-se que a "Gravidez não planejada" ( $p=0,02)$ e a "falta de apoio do pai do bebê" ( $p=$ $0,01)$ se mostraram como fatores associados ao risco para desenvolver a DPP, conforme Tabela 3.

Observa-se na Tabela 4 que, comparando os níveis de "ansiedade e depressão gestacionais" no GI e o GC, identifica-se que a porcentagem maior de mulheres com sintomas de ansiedade moderada e grave foram para as do GI (45\%), enquanto para o GC foram apenas $31 \%$. Verifica-se ainda, que ocorreu a mesma proporção de mulheres com sintomas de depressão moderada e grave na gravidez que participaram ou não do pré-natal psicológico. Destaca-se na Tabela 4 que, ao contrário do esperado, $45 \%$ das mulheres $(\mathrm{n}=13)$ do GC apresentaram sintomas de depressão aos três meses após o parto, enquanto que apenas $11 \%$ das mulheres $(n=5)$ do GI manifestaram sintomas de DPP após três meses de nascimento da criança.
A Tabela 5 indica que há associação entre os níveis de sintomas de ansiedade ( $p$-valor $=0,00015$ ) e depressão gestacionais ( $p$-valor $=0,00045$ ) com os sintomas de DPP $(p<0,05)$ para o GC.

A Tabela 6 revela que não há associação entre os sintomas de ansiedade ( $p$-valor $=0,4983)$ e depressão ( $p$-valor $=0,3023)$ apresentados na gestação com os sintomas de depressão pós-parto $(p>0,05)$, para as colaboradoras do GI.

A Tabela 7 demostra que, ao associar os indicadores considerados na literatura como fatores de proteção associados à DPP, apenas os que se mostraram associados à ausência de sintomas de DPP pelas participantes desse estudo, foram "participar do PNP" $(p=0,002)$ e o "desejar a gravidez" $(p=0,031)$.

\section{Discussão}

O alto índice de $23,68 \%$ encontrado na presente investigação reforça a necessidade apontada em vários estudos, como os de Arrais et al. (2014; 2015), Bos et al. (2013), Galvão et al. (2015), Lara et al. (2010) e Le, Perry, e Stuart (2011), sobre a importância da identificação dos sintomas iniciais que desencadeiam a DPP no puerpério, pois quanto antes se detectarem os fatores de risco e de proteção para DPP, melhor assistência poderá ser oferecida à puérpera. Desta forma, a alta prevalência de DPP encontrada reforça seu significado como problema de saúde pública, exigindo estratégias de prevenção e tratamento, como o PNP (Arrais et al., 2014; 2015, Arrais, \& Araujo, 2016). 
Tabela 1

Dados sociodemográficos e obstétrico das colaboradoras.

\begin{tabular}{|c|c|c|c|c|}
\hline & \multicolumn{2}{|c|}{ Grupo de intervenção } & \multicolumn{2}{|c|}{ Grupo controle } \\
\hline & Frequência & Porcentagem & Frequência & Porcentagem \\
\hline \multicolumn{5}{|l|}{ Estado Civil } \\
\hline Casada & 26 & $53,31 \%$ & 13 & $44,82 \%$ \\
\hline União estável & 14 & $29,78 \%$ & 6 & $20,88 \%$ \\
\hline Solteira & 6 & $12,76 \%$ & 6 & $20,88 \%$ \\
\hline Divorciada & 1 & $2,12 \%$ & 3 & $10,34 \%$ \\
\hline Viúva & 0 & $0 \%$ & 1 & $3,44 \%$ \\
\hline \multicolumn{5}{|l|}{ Escolaridade } \\
\hline Fundamental I & 1 & $2,12 \%$ & 1 & $3,44 \%$ \\
\hline Fundamental II & 5 & $10,63 \%$ & 3 & $10,34 \%$ \\
\hline Médio & 20 & $42,55 \%$ & 10 & $34,48 \%$ \\
\hline Superior & 19 & $40,42 \%$ & 13 & $44,82 \%$ \\
\hline Não informado & 2 & $4,25 \%$ & 1 & $3,44 \%$ \\
\hline \multicolumn{5}{|l|}{ Atividade ocupacional } \\
\hline Empregada & 25 & $53,19 \%$ & 20 & $68,96 \%$ \\
\hline Desempregada & 19 & $40,42 \%$ & 9 & $31,03 \%$ \\
\hline Não informaram & 3 & $6,38 \%$ & 0 & $0 \%$ \\
\hline \multicolumn{5}{|l|}{ Renda familiar } \\
\hline Até $\mathrm{R} \$ 540,00$ & 5 & $10,63 \%$ & 3 & $10,34 \%$ \\
\hline Até $\mathrm{R} \$ 1.000,00$ & 14 & $29,78 \%$ & 5 & $17,24 \%$ \\
\hline Até R $\$ 3.000,00$ & 6 & $12,76 \%$ & 6 & $20,68 \%$ \\
\hline$>\mathrm{R} \$ 4.000,00$ & 9 & $19,4 \%$ & 2 & $6,89 \%$ \\
\hline Não informaram & 13 & $27,65 \%$ & 1 & $37,93 \%$ \\
\hline \multicolumn{5}{|l|}{ Paridade } \\
\hline Primigestas & 27 & $57,11 \%$ & 16 & $55,17 \%$ \\
\hline Multigesta & 20 & $42,55 \%$ & 13 & $44,88 \%$ \\
\hline \multicolumn{5}{|l|}{ Religião } \\
\hline Católica & 22 & $46,80 \%$ & 12 & $41,37 \%$ \\
\hline Protestante & 14 & $29,78 \%$ & 8 & $27,58 \%$ \\
\hline Espírita & 4 & $8,51 \%$ & 2 & $6,89 \%$ \\
\hline Espiritualista & 3 & $6,38 \%$ & 1 & $3,44 \%$ \\
\hline Batista & 1 & $2,12 \%$ & 0 & $0 \%$ \\
\hline Luterana & 0 & $0 \%$ & 1 & $3,44 \%$ \\
\hline Testemunha de Jeová & 0 & $0 \%$ & 1 & $3,44 \%$ \\
\hline Não informou & 3 & $6,38 \%$ & 4 & $13,79 \%$ \\
\hline
\end{tabular}


Tabela 2

Teste de associação dos dados socioeconômicos e sintomas de depressão pós-parto $(\mathrm{N}=76)$.

\begin{tabular}{lcc}
\hline Dados sociodemográficos & Estatística do teste & $P$-valor \\
\hline Idade & $0,2887^{*}$ & 0,7750 \\
Religião & $6,8171^{* *}$ & 0,3528 \\
Estado civil & $6,8784^{* *}$ & 0,1259 \\
Escolaridade & $4,5924^{* *}$ & 0,3358 \\
Ocupação & $1,8025^{* *}$ & 0,1794 \\
Renda familiar & $5,0262^{* *}$ & 0,4248 \\
Paridade & $0,0000^{* *}$ & 1,0000 \\
\hline
\end{tabular}

*Teste T.

**Utilização da técnica de Monte Carlo (Q-quadrado).

Tabela 3

Teste de associação dos indicadores de fatores de risco para os sintomas de depressão pós-parto.

\begin{tabular}{lcc}
\hline Indicadores de risco & Estatística do teste & $P$-valor \\
\hline Gravidez não planejada & 0,9972 & $0,02^{*}$ \\
Falta de apoio do pai do bebê & 0,9971 & $0,01^{* *}$ \\
\hline
\end{tabular}

${ }^{*}$ Q-quadrado.

**Teste Fisher.

Tabela 4

Indicadores de saúde mental da amostra que participou do grupo controle e do grupo de intervenção.

\begin{tabular}{lcccc}
\hline \multirow{2}{*}{ Indicar de saúde mental } & Grupo Controle (n= 29) & \multicolumn{2}{c}{ Grupo de Intervenção (n=47) } \\
\cline { 2 - 5 } & $\mathrm{N}$ & $\%$ & $\mathrm{~N}$ & 21 \\
\hline Sintomas de Ansiedade Moderada e Grave Gestacional & 9 & 31,0 & 45,0 & 21,0 \\
Sintomas de Depressão Moderada e Grave Gestacional & 6 & 21,0 & 10 & 5 \\
Sintomas de depressão pós-parto & 13 & 45,0 & 11,0 \\
\hline
\end{tabular}

Tabela 5

Teste de associação entre sintomas de ansiedade e depressão gestacional com sintomas de depressão pós-parto no grupo controle.

\begin{tabular}{lcccc}
\hline Escalas & Teste & Estatística do teste & P-valor & Decisão do teste \\
\hline Níveis de ansiedade gestacional & Q-quadrado & 12,8270 & 0,0015 & $\begin{array}{c}\text { Evidências que } \\
\text { há associação }\end{array}$ \\
Níveis de depressão gestacional & Q-quadrado & 10,1310 & 0,0045 & $\begin{array}{c}\text { Evidências que } \\
\text { há associação }\end{array}$ \\
\hline
\end{tabular}

*Utilização da técnica de Monte Carlo.

Quanto aos dados socioeconômicos não terem se associado à DPP, os achados de Alharbi e Abdulghani (2014), Bos et al. (2013), Guedes et al. (2011), Nunes e Phipps (2013) e Zaconeta et al. (2013) e também não encontraram associação entre a idade materna e os sintomas de DPP. E apesar de eventos estressores aumentarem as chances de sintomas de DPP (Adams, Eberhard-Gran,Sandvik, \& Eskild, 2012; Brummelte, 
Tabela 6

Associação entre sintomas de ansiedade e depressão na gestação com sintomas de depressão pós-parto no grupo de intervenção.

\begin{tabular}{|c|c|c|c|c|}
\hline Escalas & Teste & Estatística do teste & P-valor & Decisão do teste \\
\hline Níveis de ansiedade gestacional & Q-quadrado & 2,2562 & 0,4983* & $\begin{array}{l}\text { Evidências que não } \\
\text { há associação }\end{array}$ \\
\hline Níveis de depressão gestacional & Q-quadrado & 3,5599 & $0,3023^{*}$ & $\begin{array}{l}\text { Evidências que não } \\
\text { há associação }\end{array}$ \\
\hline
\end{tabular}

*Utilização da técnica de Monte Carlo.

Tabela 7

Teste de associação dos indicadores de fatores de proteção para os sintomas de depressão pós-parto.

\begin{tabular}{lcc}
\hline Indicadores de risco & Estatística do teste & $P$ - valor \\
\hline Participou do PNP & $8,8675^{*}$ & 0,002 \\
Gravidez desejada & $0,0190^{* *}$ & 0,031 \\
\hline
\end{tabular}

*Q-quadrado.

**Teste Fisher.

\& Galea, 2010; Liu, \& Tronick, 2013; Rodrigues, \& Schiavo, 2011; Wood et al., 2010), principalmente eventos estressores em relação a problemas financeiros (Liu, \& Tronick 2013; Wood et al., 2010), pesquisas indicaram que a paridade, escolaridade e ocupação materna, não foram considerados fatores de risco para sintomas de depressão no pós-parto (Alharbi, \& Abdulghani, 2014; Bos et al., 2013; Guedes et al., 2011; Zaconeta et al., 2013), assim como na presente investigação. No tocante ao estado civil, Guedes et al. (2011) também não verificaram associação com sintomas de DPP. Entretanto, Zaconeta et al. (2013) encontraram dados que indicam que mulheres solteiras apresentam mais sintomas de DPP, quando comparada as casadas.

$\mathrm{O}$ fator de risco que mais se mostrou frequente nas respostas das participantes desse estudo foi o ter passado por intercorrências na gestação, tais como convulsões, sangramento, hematoma subcoriônico e hipertensão. A alta frequência de intercorrências na amostra estudada é inerente a gestação de alto risco, que exigiu a internação e paralisação das atividades de rotina, na referida maternidade, podendo levar a mulher a sentir medo com a possibilidade do agravamento do quadro e a incerteza sobre futuros problemas no parto e com o bebê (Brasil, 2012). Esse fator de risco físico/obstétrico está em conformidade com os estudos de Figueira et al. (2011), Urdaneta et al. (2011) e Zambaldi et al. (2010) que também verificaram que a vivência de complicações médicas na gestação aumentou as chances de desenvolver DPP.

O segundo indicador de risco mais frequente foi o nascimento via cesariana. Apesar de a Organização Mundial da Saúde (OMS) preconizar que a taxa ideal de cesáreas deveria ficar entre $10 \%$ a $15 \%$ do total de partos, a taxa de cesárea é muito superior no Brasil, com $47,8 \%$ dos nascimentos ocorrendo pela cirurgia cesariana em maternidades públicas e a alarmantes 90,8\% na rede particular (Torres et al., 2014). Entretanto, pode se pensar que o fato de ter ocorrido com frequência nessa amostra deve-se mais ao fato de serem pacientes de alto riso, o que pode ter levado a indicações de cesariana. Skari et al. (2002) concluíram que puérperas que realizaram cesarianas, sobretudo de emergência, apresentaram sinais de sofrimento psíquico relacionado à cirurgia nas primeiras semanas após o parto.

Os testes de associação mostraram que a gravidez não planejada e a falta de apoio do pai do bebê e foram associados à DPP da mãe. O estudo de Nunes e Phipps (2013) evidenciou que a presença de apoio social, em geral, reduz a frequência de mães com sintomas de DPP, identificando a falta de apoio como um elemento da DPP entre as mães. Da mesma forma, o estudo de Airosa e Silva (2013) que teve como objetivo analisar uma possível associação entre suporte social, sintomatologia depressiva, ansiosa e estresse, e a vinculação 
mãe-bebê, reforçaram que quanto maior a satisfação de apoio social, menor seriam os sintomas apontados.

Em referência à falta de apoio pai do bebê associado ao desenvolvimento da DPP, outros estudos confirmaram esse achado, como o de Guedes et al. (2011) que evidenciou que o relacionamento conjugal e ajuda insatisfatória mostraram-se como fator de risco para DPP; assim como na pesquisa de Masmoudi et al. (2014) na qual também se verificou o relacionamento marital ruim, além o baixo nível socioeconômico, dificuldade com a gravidez e a presença de antecedentes psiquiátricos como fatores de risco para DPP. As pesquisas de Al Hinai e Al Hinai (2014) e Konradt et al. (2011) também evidenciaram relação entre a percepção da falta de suporte social na gravidez e a ocorrência de depressão da mãe no período pós-parto, que encontrou associação significativa os escores de depressão e conflito com o pai da criança.

No tocante à ansiedade gestacional, os resultados mostraram que apenas as mulheres que apresentaram ansiedade leve na gestação no GC, não apresentaram maior chance de desenvolver a DPP. Já as que apresentaram de ansiedade moderada ou grave durante a gestação, de fato foram rastreadas como tendo maior risco de desenvolver a DPP. A ansiedade gestacional também foi associada à DPP nas pesquisas de Lara et al. (2010), Davey et al. (2011), Figueira et al. (2011), Rogers et al. (2013) e Garfield et al. (2015) que confirmam os achados da presente pesquisa.

Quanto à depressão gestacional, observa-se que mais da metade das mulheres do GC que apresentaram níveis de depressão leve na gestação, apresentaram maior risco de desenvolver a DPP. Já as que apresentaram depressão gestacional moderada ou grave, de fato foram rastreadas como tendo maior risco de desenvolver a DPP. Esses achados são concordantes com os resultados de Faisal-Cury e Menezes (2012), Nunes e Phipps (2013), McMahon et al. (2015) e Turkcapar et al. (2015) que também identificaram a depressão gestacional associada como um fator de risco para DPP. Ademais, esses dados confirmam a observação clínica já descrita na literatura científica em que algumas mulheres com DPP já estavam deprimidas na gestação (Aliane et al., 2011) e justifica a nova nomenclatura de depressão periparto, adotada pela American Psychological Association (APA) a partir de 2014 (APA, 2014).

Foi visto que, para as mulheres do GC, os níveis de ansiedade e depressão gestacionais estão asso- ciados com o risco de desenvolver DPP. Ou seja, as mulheres que não participaram do PNP, os níveis de ansiedade e depressão estão associados com maior chance de ter DPP. Em contrapartida, os níveis de ansiedade apresentados pelas mulheres durante a gestação, diferentemente do GC, não implicaram em um maior risco de desenvolver a DPP, mesmo nos casos de níveis moderados e graves. Esses resultados vão ao encontro do estudo realizado por Rojas et al. (2010), que verificou que um terço das gestantes têm sintomas depressivos e/ou ansiedade durante a gravidez, enquanto os valores de prevalência aumentam no pós-parto para mais de $40 \%$. Pré e DPP são altamente prevalentes e do impacto de ambos, tratadas e não tratadas de depressão perinatal, devem ser considerados no acompanhamento de mulheres grávidas deprimidas (Rojas et al., 2010).

Quanto à frequência de mulheres com algum indicador considerado de proteção para DPP foi possível identificar que o suporte familiar é o indicador dentre os fatores de proteção mais presente nas mães do estudo. Esse resultado é favorável ao que a literatura da área diz sobre a importância do apoio social no contexto da maternidade, tanto no período pré-natal e pós-natal (Airosa, \& Silva, 2013; Goodman, 2007; Nunes, \& Phipps, 2013).

Em relação à evidência de associação entre Gravidez não planejada e maior chance de DPP, esse resultado é concorde com as investigações de Castro et al. (2015), Rojas et al. (2010) e Turkcapar et al. (2015) que encontraram dados semelhantes. Rapoport e Piccinini (2006) destacaram a experiência da maternidade como um momento de sentimentos ambivalentes e, com a chegada do bebê e as consequentes situações revividas pelas mães e/ou por suas características de personalidade, os sentimentos depressivos poderiam surgir. Nesse estudo, as mães relataram sentimentos e dificuldades da experiência do ser mãe, como desespero, cansaço, medo e falta de paciência, mas consideraram seus maridos, mães e sogras como uma ajuda nos períodos mais difíceis. $\mathrm{O}$ apoio não anulou as experiências negativas, mas os autores atentaram para a possibilidade de essas terem sido amenizadas, fazendo com que as mães vivenciassem a experiência da maternidade de uma forma mais positiva.

Referente à associação entre os fatores de proteção e os sintomas de DPP, apenas os fatores "participar do PNP" e "ter desejado a gravidez" se mostraram associados à ausência de sintomas de DPP, neste 
estudo. Por mais que a gravidez seja desejada, mesmo que não planejada, como ocorreu com metade das participantes, sempre existirão ambivalência, receios e expectativas normais quanto ao bebê e às mudanças na sua vida (Rapoport, \& Piccinini, 2006). O que realmente se destaca neste fator de proteção, aliado aos fatores de proteção anteriores, é a relação sólida e de apoio com o pai do bebê, é o desejo do casal. Tal desejo não exime o aparecimento da ambivalência, mas pode fazer com que enfrentem melhor e juntos os desafios da maternidade. Outro fator de proteção que está intrinsecamente ligado à dinâmica do casal é o suporte familiar (Rapoport, \& Piccinini, 2006).

Em estudo semelhante apresente investigação, Tian Tian et al. (2012), as mulheres do grupo de intervenção foram tratadas por meio da prevenção de discussão DPP e manual conhecimento tratamento, aconselhamento, e as intervenções de aconselhamento psicológico, por telefone, visando a fatores de risco individuais, enquanto que aqueles no grupo de controle foram tratados com métodos convencionais, no grupo de intervenção e no grupo de controle. A intervenção psicológica pode reduzir a DPP, com um melhor cumprimento da função materna (Tian Tian et al., 2012), o que corrobora os achados da presente investigação e reforça a eficácia do PNP.

Também, a investigação de Collado, Saez, Favrod e Hatem (2014) que avaliou o impacto de um programa de pré-natal com base numa abordagem psicossomática à gravidez e ao parto, quanto ao risco de DPP, os resultados mostraram que a abordagem psicossomática pode ser mais útil para a população de baixa renda do que os programas de pré-natal padrão.

No estudo de Greinert e Milani (2015) que buscou identificar os fatores psicossociais que podem favorecer o desenvolvimento da DPP concluiu que são necessários programas de prevenção da DPP que levem em consideração os fatores psicossociais descritos, os quais poderiam ser desenvolvidos durante a gravidez, pois as alterações físicas, emocionais e sociais já começam a florescer na futura mãe.

A pesquisa de Kozinszky et al. (2012), que visou avaliar a eficácia de uma intervenção breve grupal e preventiva para a DPP, constatou que a intervenção com foco na psicoeducação, gestão do stress, melhorar os mecanismos de enfrentamento, bem como o desenvolvimento do apoio social pode ser eficaz na redução pós-parto sintomatologia depressiva parece reduzir significativamente o risco de DPP.
Em contrapartida, o estudo realizado por Le et al. (2011), para avaliar a eficácia de uma intervenção cognitivo-comportamental (TCC) para prevenir a DPP, revelou que intervenção CBT de baixa renda, latinas de alto risco reduziu os sintomas depressivos durante a gravidez, mas não durante o período pós-parto. Os baixos níveis de sintomas depressivos e mais baixos do que o esperado taxas de depressão clínica em ambos os grupos pode ser parcialmente devido a questões metodológicas.

\section{Conclusão}

Defende-se que o tema da pesquisa é atual e relevante, e representa uma contribuição para a área da Psicologia, especialmente àquela voltada para 0 estudo da prática da Psicologia da saúde em hospitais e instituições de saúde.

Os resultados encontrados confirmaram apenas parcialmente dos fatores de risco e proteção apontados pela literatura da área, o que leva a concluir que fatores individuais e subjetivos de cada mulher, a cultura em que está inserida, a qualidade das relações com sua rede de apoio impactam diretamente a vivência de sua maternidade.

A intervenção do PNP corroborou a literatura quanto aos efeitos deste tipo de trabalho: a diminuição o risco de desenvolver a DPP. O apoio fornecido pelo PNP não anulou os fatores de risco, mas acredita-se que esses possam ter sido amenizados, juntamente com os outros fatores de proteção, o que pode ter levado a uma experiência da maternidade mais positiva e consequentemente ao não desenvolvimento da DPP.

Apesar dos resultados relevantes para o estudo e prevenção da DPP, essa investigação apresenta algumas limitações. Uma delas se relaciona à necessidade de se ampliar a amostra, um "n" maior poderia mostrar novas associações significativas. Outra fragilidade no estudo, foi não ter acompanhado e mensurado a influência do PNP quanto à prevenção para a Depressão no período pós-parto tardio. Teria sido importante incluir na coleta de dados outros momentos de folow up ao longo do $1^{\circ}$ ano de vida do bebê; já que o tempo da coleta de amostra do estudo foi limitado a três meses do pós-parto, em função das várias dificuldades de encontrar as mães no puerpério. Seria interessante também, proceder a outras análises estatísticas mais abrangentes para medir fator de risco e proteção, pois o Q-quadrado só verifica associação ou comparação 
entre as variáveis, mas não permite identificar risco ou proteção como os testes de regressão, por exemplo. Sugere-se ainda, que estudos futuros utilizem a metodologia qualitativa ou mista para avaliação das participantes quanto à satisfação com o PNP, bem como a sua inter-relação com outros fatores de risco e proteção, e não só a quantitativa, como foi a base da análise metodológica da presente investigação.

São necessários mais estudos sobre essa temática, e de ampliar pesquisas sobre a participação psicoprofilática da Psicologia clínica e da saúde no processo do ciclo gravídico-puerperal e em serviços de obstétrica, corroborando para expansão dessa prática não apenas no contexto hospitalar diante de gestações ou no momento do parto, mas que as mulheres e famílias possam ter acesso ao acompanhamento psicológico durante essa fase transitória da vida.

Por fim, os resultados confirmam a importância do conhecimento dos fatores de risco e de proteção da DPP para o planejamento e execução de ações preventivas ainda na fase pré-natal, para viabilizar a promoção do cuidado integral a gestante e puérpera, desprendendo-se da exclusividade do modelo biomédico e concretizando as diretrizes das políticas públicas mais recentes e os avanços nas áreas de Psicologia Clínica e da Saúde e até mesmo da Obstetrícia e Saúde da Mulher.

\section{Referências}

Adams, S, Eberhard-Gran M, Sandvik A. \& Eskild A. (2012). Mode of delivery and postpartum emotional distress: a cohort study of 55814 women. Journal of Obstetrics \& Gynaecology,119(3), 298-305. https://doi.org/10.1111/j. 1471-0528.2011.03188.x

Airosa, S., \& Silva, I. (2013). Associação entre vinculação, ansiedade, depressão, stresse e suporte social na maternidade. Psicologia, Saúde \& Doenças, 14(1), 64-77. Recuperado de http://www.scielo.mec.pt/scielo.php?scrip$\mathrm{t}=\mathrm{sci}$ arttext\&pid=S1645-00862013000100005\&lng=pt\&tlng=pt

Alharbi, A. A., \& Abdulghani, H. M. (2014).Risk factors associated with postpartum depression in the Saudi population. Neuropsychiatric Disordes Treatement, 10, 311-316. https://doi.org/10.2147/NDT.S57556

Al Hinai, F. I., \& Al Hinai, S. S. (2014). Prospective study on prevalence and risk factors of postpartum depression in Al-Dakhliya Governorate in Oman. Oman Medical Journal, 29(3),198-202. https://doi.org/10.5001/omj.2014.49

Aliane, P. P., Mamede, M. V., \& Furtado, E. F. (2011). Revisão sistemática sobre fatores de risco associados à depressão pós-parto. Psicologia em Pesquisa, 5(2), 146-155. Recuperado de http://www.uff.br/psicologiaempesquisa/ files/2011/12/v5n2a07.pdf

American Psychiatric Association - APA. (2014). Diagnostic and statistical manual of mental disorders - DSM-5 (5th ed.). Washington, DC: American Psychiatric Association.

Arrais, A. R., \& Araujo, T. C. C. F. (2016). Pré-Natal Psicológico: perspectivas para atuação do psicólogo em Saúde Materna no Brasil. Revista da SBPH, 19(1), 103-116. Recuperado de http://pepsic.bvsalud.org/scielo.php?scrip$\mathrm{t}=\mathrm{sci} \_$arttext\&pid=S1516-08582016000100007\&lng=pt\&tlng=pt

Arrais, A. R., Lordello, S. R., \& Cavados, G. C. F. (2015). O pré-natal psicológico como fator de proteção à depressão pós-parto. In S. G. Murta, C. França, L. K. B Santos, \& L. Polejack. (Eds.), Prevenção e promoção em saúde mental: Fundamentos, planejamento e estratégias de intervenção (pp. 601-621). Novo Hamburgo, RS: Sinopsys.

Arrais, A. R., Mourão, M. A., \& Fragalle, B. (2014). O pré-natal psicológico como programa de prevenção à depressão pós-parto. Saúde \& Sociedade, 23(1), 251-264. https://doi.org/10.1590/S0104-12902014000100020

Beck, A. T., \& Steer, R. A. (1993). Manual for the Beck Depression Inventory. San Antonio, TX: Psychological Corporation.

Beck, A. T., Steer, R. A., \& Brown, G. K. (1996). Manual for the Beck Depression Inventory-II. San Antonio, TX: Psychological Corporation.

Bhandari, S., Bullock, L. F., Bair-Merritt, M., Rose, L., Marcantonio, K., Campbell, J. C., \& Sharps, P. (2012). Pregnant women experiencing IPV: Impact of supportive and non-supportive relationships with their mothers and other supportive adults on perinatal depression: a mixed methods analysis. Issues in Mental Health Nursing, 33(12), 827-837. https://doi.org/10.3109/01612840.2012.712628

Bortoletti, F. F. (2007). Psicodinâmica do ciclo gravídico puerperal. In F. F. Bortoletti (Eds.), Psicologia na prática obstétrica: Abordagem interdisciplinar (pp. 21-31). Barueri, SP: Manole. 
Bos, S. C., Macedo, A., Marques, M., Pereira, A. T., Maia, B. R., Soares, M. J. et al. (2013). Is positive affect in pregnancy protective of postpartum depression? Revista Brasileira de Psiquiatria, 35(1), 5-12. https://doi.org/10. 1016/j.rbp.2011.11.002

Bottino, M. N. (2011). Aspectos evolutivos da depressão pós-parto e fatores associados (Tese). Universidade do Estado do Rio de Janeiro, Rio de Janeiro, RJ, Brasil.

Brasil (2012). Ministério da Saúde. Atenção ao pré-natal de baixo risco. Brasília, DF: o autor.

Brummelte, S., \& Galea, L.A. (2010). Chronic corticosterone during pregnancy and postpartum affects maternal care, cell proliferation and depressive-like behavior in the dam.Hormones and Behavior, 58(5), 769-779. httpS:// doi.org/10.1016/j.yhbeh.2010.07.012

Calvetti, P. U., Muller, M. C., \& Nunes, M. L. T. (2007). Psicologia da saúde e psicologia positiva: perspectivas e desafios. Psicologia, Ciência \& Profissão, 27(4), 706-717. https://doi.org/10.1590/S1414-98932007000400011

Cantillino, A., Zambaldi, C. F., Albuquerque, T. L. C., \& Sougey, E. B. (2010). Depressão pós-parto em Recife - Brasil: Prevalência e associação com fatores biossociodemográficos. Jornal Brasileiro de Psiquiatria, 59(1), 1-9. https:// doi.org/10.1590/S0047-20852010000100001

Carlesso, J. P. P., Souza, A. P. R., \& Moraes, A. B. (2014). Análise da relação entre depressão materna e indicadores clínicos de risco para o desenvolvimento infantil. Revista CEFAC, 16(2), 500-510. https:// doi.org/10.1590/19820216201418812

Castro, F., Place, J. M., Billings, D.L., Rivera, L., \& Frongillo, E. A. (2015). Risk profiles associated with postnatal depressive symptoms among women in a public sector hospital in Mexico: The role of sociodemographic and psychosocial factors. Archive of Women's Mental Health, 18(3), 463-471. https://doi.org/10.1007/s00737-014-0472-1

Collado, M. A. O., Saez, M., Favrod, J., \& Hatem, M. (2014). Antenatal psychosomatic programming to reduce postpartum depression risk and improve childbirth outcomes: A randomized controlled trial in Spain and France. BioMed Central Pregnancy \& Childbirth,14, 22. https:// doi.org/10.1186/1471-2393-14-22

Conselho Federal de Psicologia - CFP. (2005). Código de ética professional do psicólogo. Brasília, DF: o autor.

Cox, J., \& Holden, J. (2003). Perinatal mental health: A guide to the Edinburgh Postnatal Depression Scale (EPDS). London: Royal College of Psychiatrists, Gaskell.

Dantas, M. M. C. D., Araújo, P. C. B., Paulino, D. S., \& Maia, E. M. C. (2012). Avaliação do apoio social e de sintomas depressivos em mães de bebês prematuros hospitalizados. Psicologia em Revista,18(1), 90-106. https://doi. org/10.5752/P.1678-9563.2012v18n1p90

Davey, H. L., Tough, S. C., Adair, C. E., \& Benzies, K. M. (2011). Risk factors for sub-clinical and major postpartum depression among a community cohort of canadian women. Maternal Child Health Journal, 15(7), 866-875. https://doi.org/10.1007/s10995-008-0314-8

Ding, T., Wang, D.X., Qu, Y., Chen, Q., \& Zhu, S.N. (2014). Epidural labor analgesia is associated with a decreased risk of postpartum depression: a prospective cohort study. Anesthesia \& Analgesia, 119(2), 383-92. https:/doi. org/10.1213/ANE.0000000000000107

Dois, A. Uribe, C., Villarroel, L., Contreras, A. (2012). Factores de riesgo asociados a síntomas depresivos pós-parto en mujeres de bajo riesgo obstétrico atendidas em el sistema público. Revista Médica de Chile, 140(6), 719-725. https://doi.org/10.4067/S0034-98872012000600004

El-Hachem, C., Rohayem, J., KhalilEmail, R. B., Richa, S., Kesrouani, A., Gemayel, R. et al. (2014). Early identification of women at risk of postpartum depression using the Edinburgh Postnatal Depression Scale in a sample of Lebanese women. BioMed Central Psychiatry, 14, 242-247. https://doi.org/10.1186/s12888-014-0242-7

Faisal-Cury, A., \& Menezes, P. R. (2012). Depressão antenatal prediz fortemente depressão pós-parto na atenção básica à saúde. Revista Brasileira de Psiquiatria, 34, 446-450. https://doi.org/1016/j.rbp.2012.01.003

Figueira, P. G., Diniz, L. M., \& Silva Filho, H. C. (2011). Características demográficas e psicossociais associadas à depressão pós-parto em uma amostra de Belo Horizonte. Revista de Psiquiatria do Rio Grande do Sul, 33(2), 71-75. https://doi.org/10.1590/S0101-81082011005000009

Flores, M. R., Souza, A. P. R., Moraes, A. B., \& Beltrami, L. (2013). Association between risk indicators for child development and maternal emotional state. Revista CEFAC, 15(2), 348-360. https://doi.org/10.1590/S151618462012005000046 
Galvão, A. C. C., Silva Júnior, F. J. G., Lima, L. A. A, \& Monteiro, C. F. S. (2015). Prevalência de depressão pós-parto e fatores associados: Revisão sistemática. Revista OnFacema, 1, 54-58. Recuperado de http://www.facema.edu.br/ ojs/index.php/ReOnFacema/article/view/3

Garfield, L., Holditch-Davis, D., Carter, C. S., McFarlin, B. L., Schwertz, D., Seng, J. S. et al. (2015). Risk factors for postpartum depressive symptoms in low-income women with very low-birth-weight infants. Advances in Neonatal Care Journal, 15(1), 3-8. https://doi.org/10.1097/ANC.0000000000000131

Goodman, S. H. (2007). Depression in mothers. Annual Review of Clinical Psychology, 3, 107-135. https://doi. org/10.1146/annurev.clinpsy.3.022806.091401

Greinert, B. R. M., \& Milani, R. G. (2015). Depressão pós-parto: Uma compreensão psicossocial. Revista Psicologia: Teoria e Prática, 17(1), 26-36. https://doi.org/10.15348/1980-6906/psicologia.v17n1p26-36

Guedes, A. C. E., Kami, C. T., Cavalli, L. K. V., Nicolaou, S. K., Hess, V. B., Maluf, E. M. C. P. (2011). Depressão pós-parto: Incidência e fatores de risco associados. Revista de Medicina, 90(3), 149-154. https://doi.org/10.11606/ issn.1679-9836.v90i3p149-154

Janssen, P. A., Heaman, M. I.,Urquia, M. L., O'Campo, P. J., Thiessen, K. R. (2011). Experiences of violence before and during pregnancy and adverse pregnancy outcomes: an analysis of the Canadian Maternity Experiences Survey. BioMed Central Pregnancy \& Childbirth, 11, 42. https://doi.org/10.1186/1471-2393-11-42

Kathree, T., Selohilwe, O. M., Bhana, A. \& Petersen, I. (2014). Perceptions of postnatal depression and health care needs in a South African sample: the "mental" in maternal health care. BMCWomen's Health, 14, 140. https://doi. org/10.1186/s12905-014-0140-7

Kim, T. H. M., Connolly, J. A., \& Tamim, H. (2014). The effect of social support around pregnancy on postpartum depression among canadian teen mothers and adult mothers in the maternity experiences survey. BioMed Central Pregnancy \& Childbirth, 14, 162. https://doi.org/10.1186/1471-2393-14-162

Kinsey, C. B., Baptiste-Roberts, K., Zhu, J., \& Kjerulff, K. H. (2014). Effect of miscarriage history on maternal-infant bonding during the first year postpartum in the First Baby Study: a longitudinal cohort study. BMC Women's Health, 14, 83. https://doi.org/10.1186/1472-6874-14-83

Konradt, C. E., Silva, R. A., Jansen, K., Vianna, D. M., Quevedo, L. A., Souza, L. D. M. et al. (2011). Depressão pós-parto e percepção de suporte social durante a gestação. Revista de Psiquiatria do Rio Grande do Sul, 33(2), 76-79. https://doi.org/10.1590/S0101-81082011005000010

Kozinszky, Z.,Dudas, R. B., Devosa, I., Csatordai, S., Tóth, E., Szabó, D. et al. (2012). Can a brief antepartum preventive group intervention help reduce postpartum depressive symptomatology? Psychotherapy and Psychosomatics, 81(2), 98-107. https://doi.org/10.1159/000330035

Lara, M. A., Navarro, C., Navarrete, L., \&Yle, H. (2010). Tasas de retención y variables que lapredicen em um estúdio aleatorio controlado longitudinal para prevenir ladepresiónposparto. Salud Mental, 33(5), 429-436. Recuperado de http://www.scielo.org.mx/scielo.php?script=sci_arttext\&pid=S0185-33252010000500007

Le, H. N., Perry, D. F., \& Stuart, E. A. (2011).Randomized controlled trial of a preventive intervention for perinatal depression in high-risk latinas. Journal of Consulting and Clinical Psychology,79(2), 135-141. https://doi. org/10.1037/a0022492

Lima, M. O. P., \& Tsunechiro, M. A. (2008). Repercussões materno-fetais da depressão na gravidez: Uma revisão sistemática. O Mundo da Saúde São Paulo, 32(4), 530-536. Retirado de http://www.saocamilo-sp.br/pdf/mundo_ saude/65/15_Repercussoes_baixa.pdf

Liu, C. H., \& Tronick, E. (2013).Re-conceptualising prenatal life stressors in predicting post-partum depression: Cumulative-, specific-, and domain-specific approaches to calculating risk. Paediatric and Perinatal Epidemiology, 27(5), 481-490. https://doi.org/10.1111/ppe.12072

Lucci, T. K., Otta, E., David, V. F., \& Chelini, M.O.M. (2016). Depressão materna e concentração de cortisol de recém-nascidos em uma amostra brasileira. Revista Psico, 47(2), 140-147. 10. https://doi.org/10.15448/19808623.2016.2.23655

Martín-Santos, R., Gelabert, E.,Subirà, S., Zotes, G., Langorh, A., K., Jover, M. et al. (2012). Is neuroticism a risk for postpartum depression? Psychological Medicine, 42(7), 1559-1565. http://dx.doi.org/10.1017/S0033291712000712 
Masmoudi, J.,Charfeddine, F., Trabelsi, S., Feki, I., Ayad, B., Guermazi, M. et al. (2014). Postpartum depression: Prevalence and risk factors: A prospective study concerning 302 tunisian parturients. Tunis Medicine, 92(10), 615-621.

McMahon, C. A., Boivin, J., Gibson, F. L., Hammarberg, K., Wynter, K., \& Fisher, J. R. (2015). Older maternal age and major depressive episodes in the first two years after birth: Findings from the parental age and transition to parenthood Australia (PATPA) study. Journal of Affective Disorders, 175, 454-462. https://doi.org/10.1016/j.jad.2015.01.025

Miller, L. J., \& La Russo, E. M. (2011). Preventing postpartum depression. Psychiatric Clinics of North America, 34(1), 53-65. https:// doi.org/10.1016/j.psc.2010.11.010

Miranda, A. M., Soares, C. N., Moraes, M. L., Fossaluza, V., Serafim, P. M., \& Mello, M. F. (2012). Healthy maternal bonding as a resilience factor for depressive disorder. Psychology \& Neuroscience, 5(1), 21-25. https://doi. org/10.3922/j.psns.2012.1.04

Morais, M. L. S., Fonseca, L. A. M., David, V. F., Viegas, L. M., \& Otta, E. (2015). Fatores psicossociais e sociodemográficos associados à depressão pós-parto: Um estudo em hospitais público e privado da cidade de São Paulo. Estudos de Psicologia, 20(1), 40-49. http://dx.doi.org/10.5935/1678-4669.20150006

Nunes, A. P., \& Phipps, M. G. (2013). Postpartum depression in adolescent and adult mothers: comparing prenatal risk factors and predictive models. Maternal and Child Health Journal, 17(6), 1071-1079. https://doi.org/10.1007/ s10995-012-1089-5

Okun, M. L., Luther, J., Prather, A. A., Perel, J. M., Wisniewski, S., \&Wisner, K. L. (2011). Changes in sleep quality, but not hormones predict time to postpartum depression recurrence. Journal of Affective Disorders, 130(3), 378-384. http://dx.doi.org/10.1016/j.jad.2010.07.015

PoojaBansil, M. P. H., Kuklina E. V., Meikle, S. F., Posner S. F., Kourtis,A. P., Sascha, R. E. et. al. (2010). Maternal and fetal outcomes among women with depression. Journal of Women`s Helth (Larchmt), 19(2), 229-234. https://doi. org/10.1089/jwh.2009.1387

Rapoport, A., \& Piccinini, C. A. (2006). Apoio social e experiência da maternidade. Revista Brasileira de Crescimento Desenvolvimento Humano, 16(1), 85-96. Recuperado de http://pepsic.bvsalud.org/scielo.php?pi$\mathrm{d}=\mathrm{S} 0104-12822006000100009 \&$ script $=$ sci_abstract

Rodrigues, O. M. P. R., \& Schiavo, R. A. (2011). Stress na gestação e no puerpério: uma correlação com a depressão pós-parto. Revista Brasileira de Ginecologia e Obstetrícia, 33(9), 252-257. https://doi.org/10.1590/S010072032011000900006

Rodríguez, I. C., Pineda, M. R., Álvarez, T. A., \& Rojas, L. Q. (2013). Factores de riesgo asociados a la prematuridade em recién nacidos de madres adolescentes. Revista Obstetricia \& Ginecolgia de Venezuela, 73(3),157-170.

Rogers, C. E., Kidokoro, H., Wallendorf, M., \& Inder, T. E. (2013). Identifying mothers of very preterm infants at-risk for postpartum depression and anxiety prior to discharge. Jornal of Perinatology, 33(3), 171-176. http://dx.doi. org/10.1038/jp.2012.7

Rohitkumar, V., Kamal, E., Vipin, G., Kuriakose, J., Nair, G., Newsom, C., \& Bates, J. (2014). Postpartum depression in mothers of infants in neonatal intensive care unit: Risk factors and management strategies. American Journal of Perinatology, 31(5),425-434. https://doi.org/1010.1055/s-0033-1352482

Rojas, G., Fritsch, R., Guajardo, V. Rojas, F., Barroilhet, S., \& Jadresic, E. (2010). Caracterización de madres deprimidas em el pos parto. Revista Médica de Chile, 138(5), 536-542. https:// doi.org/10.4067/S0034-98872010000500002

Santos, M. F. S., Martins, F. C., \& Pasquali, L. (1999). Escala de auto-avaliação de DPP: estudo no Brasil. Revista de Psiquiatria Clínica, 26(2), 90-95.

Saraiva, E. R. A., \& Coutinho, M. P. L. (2008). O sofrimento psíquico no puerpério: um estudo psicológico. Revista Mal-Estar e Subjetividade, 8(2), 505-527. Recuperado de http://www.revispsi.uerj.br/v8n3/artigos/html/v8n3al4.html

Silva, R., Jansen, K., Souza, L., Quevedo, L., Barbosa, L., Moraes, I., \& Pinheiro, R. (2012). Fatores sociodemográficos de risco de depressão perinatal: um estudo populacional no sistema público de cuidados de saúde. Revista Brasileira de Psiquiatria, 34(2), 143-148. https:/ / doi.org/10.1590/S1516-44462012000200005

Skari, H., Skreden, M., Malt, U.F., Dalholt, M., Ostensen, A.B., Egeland, T. \& Emblem,R. (2002).Comparative levels of psychological distress, stress symptoms, depression and anxiety after childbirth: A prospective population-based study of mothers and fathers. Journal of Obstetrics \& Gynaecology, 109(10),1154-1163. https://doi.org/10.1016/ S1470-0328(02)00968-0 
Sylvén, S. M., Ekselius, L., Sundström-Poromaa, I., \& Skalkidou, A. (2013). Premenstrual syndrome and dysphoric disorder as risk factors for postpartum depression.Acta Obstetricia et Gynecologica Scandinavica, 92(2), $178-184$. https://doi.org/ 10.1111/aogs.12041

Theme Filha, M. M., Ayers S., Gama S. G., \& Leal, M. C. (2016). Factors associated with postpartum depressive symptomatology in Brazil: The birth in Brazil national research study, 2011/2012. Journal of Affective Disorders, 194, 159-167. https://doi.org/10.1016/j.jad.2016.01.020

Tian Tian, T., Li, Y., Xie, D., Shen, Y., Ren, J., Wu, W., \& Tian, H. (2012). Clinical features and risk factors for post-partum depression in a large cohort of Chinese women with recurrent major depressive disorder. Journal of Affective Disorders, 136(3), 983-987. https://doi.org/10.1016/j.jad.2011.06.047

Thiollent, M. (2008). Metodologia da pesquisa-ação. 15a ed. São Paulo, SP: Cortez

Torres, J. A., Domingues, R. M. S. M., Sandall, J., Hartz., Z., G., Nogueira, S. G., Theme Filha, M. M. et al. (2014).Cesariana e resultados neonatais em hospitais privados no Brasil: estudo comparativo de dois diferentes modelos de atenção perinatal. Cadernos de Saúde Pública, 30(1), S220-S231. https://doi.org/10.1590/0102-311X00129813

Turkcapar, A. F., Kadığlu, N., Aslan, E.,Tunc, S., Zayıfoğlu, M., \& Mollamahmutoğlu, L. (2015). Sociodemographic and clinical features of postpartum depression among Turkish women: A prospective study. BioMed Central Pregnancy \& Childbirth, 15, 108. https://doi.org/10.1186/s12884-015-0532-1

Urdaneta M., Rivera, J. S., García, A. I., Guerra, J., Mery, V., Nasser, B. Z., \& Contreras, A. B. (2011). Factores de riesgo de depresión pos parto en puérperas venezolanas valoradas por medio de la escala de Edimburgo. Revista Chilena de Obstetricia y Ginecología, 76(2), 102-112. https://doi.org/10.4067/S0717-75262011000200007

Vasconcellos, A. J. A., \& Teng, C.T. (2010) Psiquiatria perinatal. São Paulo, SP: Atheneu

Wood, S.M., Melville, J. L., Yuqing Guo, M.P.H., Ming-Yu Fan, M.S.N., \& Gavin, A. M.S.W. (2010). Psychosocial stress during pregnancy. American Journal of Obstetrics and Gynecology, 202(1), 61-67. https://doi.org/10.1016/j. ajog.2009.07.041

Wu, M., Li, X.,Feng, B., Wu, H., Qiu, C., \& Zhang, W. (2014). Poor sleep quality of third-trimester pregnancy is a risk factor for postpartum depression. Medical Science Monitor, 20, 2740-2745. https://doi.org/10.12659/ MSM.891222

Zaconeta, A. M., Queiroz, I. F., Amato, A. A., Motta, L. D., \& Casulari, L. A. (2013). Depression with postpartum onset: A prospective cohort study in women undergoing elective cesarean section in Brasilia, Brazil. Revista Brasileira de Ginecologia e Obstetrícia, 35(3), 130-135. https://doi.org/10.1590/S0100-72032013000300007

Zambaldi, C. F., Cantillino, A., Sougey, E. B., \& Rennó Jr. (2010). Transtornos psiquiátricos no pós-parto. Revista de Psiquiatria Clínica, 37(6), 278-284. https:// doi.org/10.1590/S0101-60832010000600006

\section{Alessandra da Rocha Arrais}

Doutora e Pós-Doutora pela Programa de Pós-graduação em Psicologia Clínica e Cultura do Departamento de Psicologia da Universidade de Brasília (UnB), Brasília - DF. Brasil. Docente permanente do Programa de Mestrado Profissional em Ciências para a Saúde da Escola Superior de Ciências da Saúde (ESCS), Brasília-DF. Brasil. Psicóloga Hospitalar da Secretaria de Saúde do Distrito Federal (SES-DF), Brasília - DF. Brasil. Pesquisadora da Fundação de Apoio a Pesquisa do Distrito Federal (FAP-DF) e pesquisadora colaboradora da UnB e da Universidade Católica de Brasília (UCB), Brasília - DF. Brasil.

E-mail: alearrais@gmail.com

\section{Tereza Cristina Cavalcanti Ferreira de Araujo}

Psicóloga graduada pela Universidade de Brasília (UnB), Brasília - DF. Brasil. Doutora pela Université de Paris X, Nanterre. França. Pós-doutora pela Unesco, França. Professora do Departamento de Psicologia Clínica da UnB. Orientadora de Mestrado e de Doutorado no Programa de Psicologia Clínica e Cultura e no Programa de Bioética da UnB. Pesquisadora do Conselho Nacional de Desenvolvimento Científico e Tecnológico e Líder do Grupo de Pesquisa "Psicologia da Saúde e Desenvolvimento (CNPq)".

E-mail: araujotc@unb.br 


\section{Rafaela de Almeida Schiavo}

Psicóloga. Mestre pela Universidade Estadual Paulista (Unesp), Bauru - SP. Brasil. Doutora pela Universidade Estadual Paulista (Unesp), Botucatu - SP. Brasil. Docente da Universidade Paulista (Unip), Bauru - SP. Brasil. Docente do Instituto Municipal de Ensino Superior de São Manuel (IMESSM), Americana - SP. Brasil.

E-mail: rafaela.schiavo@gmail.com

Endereço para correspondência:

Alessandra da Rocha Arrais, Escola Superior de Ciências da Saúde - Setor Médico Hospitalar Norte -Conjunto A Bloco 01 - Edifício Fepecs - Asa Norte, Brasília - DF, 70710-907

Recebido 02/09/2016

Reformulado 10/05/2017

Aprovado 03/08/2017

Received 09/02/2016

Reformulated $05 / 10 / 2017$

Approved 08/03/2017

Recibido 02/09/2016

Reformulado 10/05/2017

Aceptado 03/08/2017

Como citar: Arrais, A. R., Araujo, T. C. C. F., \& Schiavo, R. A. (2018). Fatores de risco e proteção associados à depressão pós-parto no pré-natal psicológico. Psicologia: Ciência e Profissão, 38(4), 711-729. https://doi.org/10.1590/1982-3703003342016

How to cite: Arrais, A. R., Araujo, T. C. C. F., \& Schiavo, R. A. (2018). Risk factors and protection associated with postpartum depression in psychological prenatal care. Psicologia: Ciência e Profissão, 38(4), 711-729. https://doi.org/10.1590/1982-3703003342016

Cómo citar: Arrais, A. R., Araujo, T. C. C. F., \& Schiavo, R. A. (2018). Factores de riesgo y protección asociados a la depresión post-parto en el pre-natal psicológico. Psicologia: Ciência e Profissão, 38(4), 711-729. https://doi.org/10.1590/1982-3703003342016 